\title{
VARIABLE TRANSFORMATIONS AND GAUSS-LEGENDRE QUADRATURE FOR INTEGRALS WITH ENDPOINT SINGULARITIES
}

\author{
AVRAM SIDI
}

\begin{abstract}
Gauss-Legendre quadrature formulas have excellent convergence properties when applied to integrals $\int_{0}^{1} f(x) d x$ with $f \in C^{\infty}[0,1]$. However, their performance deteriorates when the integrands $f(x)$ are in $C^{\infty}(0,1)$ but are singular at $x=0$ and/or $x=1$. One way of improving the performance of Gauss-Legendre quadrature in such cases is by combining it with a suitable variable transformation such that the transformed integrand has weaker singularities than those of $f(x)$. Thus, if $x=\psi(t)$ is a variable transformation that maps $[0,1]$ onto itself, we apply Gauss-Legendre quadrature to the transformed integral $\int_{0}^{1} f(\psi(t)) \psi^{\prime}(t) d t$, whose singularities at $t=0$ and/or $t=1$ are weaker than those of $f(x)$ at $x=0$ and/or $x=1$. In this work, we first define a new class of variable transformations we denote $\widetilde{\mathcal{S}}_{p, q}$, where $p$ and $q$ are two positive parameters that characterize it. We also give a simple and easily computable representative of this class. Next, by invoking some recent results by the author concerning asymptotic expansions of Gauss-Legendre quadrature approximations as the number of abscissas tends to infinity, we present a thorough study of convergence of the combined approximation procedure, with variable transformations from $\widetilde{\mathcal{S}}_{p, q}$. We show how optimal results can be obtained by adjusting the parameters $p$ and $q$ of the variable transformation in an appropriate fashion. We also give numerical examples that confirm the theoretical results.
\end{abstract}

\section{INTRODUCTION}

Consider the problem of evaluating finite-range integrals of the form

$$
I[f]=\int_{0}^{1} f(x) d x
$$

by the $n$-point Gauss-Legendre quadrature rule

$$
G_{n}[f]=\sum_{i=1}^{n} w_{n i} f\left(x_{n i}\right),
$$

where $x_{n i}$ are the abscissas [the zeros of $P_{n}(2 x-1)$, where $P_{n}(x)$ is the $n$th Legendre polynomial] and $w_{n i}$ are the corresponding weights. Also, let

Received by the editor March 3, 2008 and, in revised form, July 28, 2008.

2000 Mathematics Subject Classification. Primary 40A25, 41A60, 65B15, 65D30, 65D32.

Key words and phrases. Variable transformations, Gauss-Legendre quadrature, singular integrals, endpoint singularities, asymptotic expansions, Euler-Maclaurin expansions.

(C)2009 American Mathematical Society Reverts to public domain 28 years from publication 


$$
E_{n}[f]=I[f]-G_{n}[f]
$$

denote the error in this approximation.

When $f \in C^{\infty}[0,1]$, the error $E_{n}[f]$ tends to zero faster than any negative power of $n$, that is, $E_{n}[f]=o\left(n^{-\mu}\right)$ as $n \rightarrow \infty$ for every $\mu>0$. In particular, when $f(z)$ is analytic in an open set that contains the interval $[0,1]$ in its interior, there holds $E_{n}[f]=O\left(e^{-\sigma n}\right)$ as $n \rightarrow \infty$ for some $\sigma>0$. See Davis and Rabinowitz [2, p. 312].

When $f(x)$ has integrable singularities at the endpoints $x=0$ and/or $x=1$, Gauss-Legendre quadrature rules $G_{n}[f]$ can be used to approximate $I[f]$ since these rules are of the open type, that is, their abscissas $x_{n i}$ are all in $(0,1)$, hence different from $x=0$ and $x=1$. This is known as "avoiding the singularity" in numerical quadrature; see [2, p. 93]. In the presence of endpoint singularities, however, the error $E_{n}[f]$ tends to zero slowly, its rate of decay depending on the strength of the singularities; the stronger the singularities, the slower the rate of convergence of $E_{n}[f]$ to zero. For example, when $f(x)=x^{\alpha} g(x)$, with $\Re \alpha>-1$ but $\alpha \neq 0,1, \ldots$, and $g \in C^{\infty}[0,1]$ and $g(0) \neq 0$, it is known that $E_{n}[f]=O\left(n^{-2 \alpha-2}\right)$ as $n \rightarrow \infty$. See [2, p. 313]. Clearly, if $\Re \alpha>0, f(x)$ is differentiable $\lfloor\Re \alpha\rfloor$ times at $x=0$ and hence on $[0,1]$. Consequently, if $\Re \alpha$ is sufficiently large, $f(x)$ is differentiable a sufficient number of times on $[0,1]$, and the Gauss-Legendre quadrature provides highly accurate approximations $G_{n}[f]$ to $I[f]$ even with small $n$. In case $\Re \alpha$ is small, we can approximate $I[f]$ with high accuracy by first weakening the singularity at $x=0$ via a suitable variable transformation and next applying Gauss-Legendre quadrature to the resulting transformed integral.

For future reference and to set some of the notation to be used later, we give the technical description of this approach at this point. Let $x=\psi(t)$ be a variable transformation that maps $[0,1]$ unto itself, that is,

$$
\psi \in C^{1}[0,1] ; \quad \psi(0)=0, \quad \psi(1)=1 ; \quad \psi^{\prime}(t)>0 \quad \text { for } t \in(0,1) .
$$

Letting $x=\psi(t)$ in (1.1), we obtain the transformed integral

$$
I[f]=\int_{0}^{1} \widehat{f}(t) d t=I[\widehat{f}] ; \quad \widehat{f}(t)=f(\psi(t)) \psi^{\prime}(t) .
$$

Following this transformation, we apply the Gauss-Legendre quadrature to the integral $I[\widehat{f}]$ and obtain good approximations to $I[f]$. Thus, the $n$-point GaussLegendre quadrature rule now gives

$$
G_{n}[\widehat{f}]=\sum_{i=1}^{n} w_{n i} \widehat{f}\left(x_{n i}\right)=\sum_{i=1}^{n} w_{n i} f\left(\psi\left(x_{n i}\right)\right) \psi^{\prime}\left(x_{n i}\right) \equiv \widehat{G}_{n}[f] .
$$

The error in $\widehat{G}_{n}[f]$ is

$$
\widehat{E}_{n}[f]=I[f]-\widehat{G}_{n}[f] .
$$

To see what can be achieved by this approach, let us consider the case in which $f(x)=x^{\alpha} g(x)$, with $\Re \alpha>-1$ but $\alpha \neq 0,1, \ldots$, and $g \in C^{\infty}[0,1]$. Let us consider a variable transformation $\psi(t)$, such that $\psi \in C^{\infty}[0,1]$ and $\psi^{\prime}(t)=O\left(t^{s}\right)$ as $t \rightarrow 0$, hence $\psi(t)=O\left(t^{s+1}\right)$ as $t \rightarrow 0, s$ being a positive integer. Then, we have that $\widehat{f}(t)=O\left(t^{\omega}\right)$ as $t \rightarrow 0+$, where $\omega=\alpha+(\alpha+1) s$. Thus, $\widehat{f}(t)=t^{\omega} \widehat{g}(t)$, where $\widehat{g} \in C^{\infty}[0,1]$. Because $\Re \alpha+1>0$, by choosing $s$ sufficiently large, we can achieve a sufficiently large value for $\Re \omega$. As a result, we can achieve very high accuracy by using $\widehat{G}_{n}[f]$, as explained above. 
This approach, with $\psi \in C^{\infty}[0,1]$, was used in the works of Johnston [4], 5] and Monegato and Scuderi [7. In [4] and [5], the integrand $f(x)$ is assumed to be singular only at one of the endpoints, whereas in [7, it is allowed to have singularities at both $x=0$ and $x=1$. An analogous approach to the computation of integrals $I[f]=\int_{0}^{1} f(x) d x$, where the functions $f(x)$ have only one (integrable) singularity in $(0,1)$, the interior of the interval of integration, but are regular at the endpoints $x=0$ and $x=1$, was used by Monegato and Sloan 8 . These authors use a polynomial variable transformation that causes the integrand to be continuously differentiable on the whole interval $[0,1]$ as many times as required, and this enables Gauss-Legendre quadrature to produce very good results.

In this work, we focus on the computation of integrals $I[f]$ of functions $f(x)$ that are in $C^{\infty}(0,1)$ but have algebraic singularities at one or both of the endpoints $x=0$ and $x=1$. We adopt the approach described via (1.4)-(1.7), whereby Gauss-Legendre quadrature is applied after performing a variable transformation. Our approach has two novel features:

(i) Unlike those used in [4, [5, and [7, the variable transformations $\psi(t)$ we use are not necessarily in $C^{\infty}[0,1]$ but only in $C^{\infty}(0,1)$, and they can be singular at $t=0$ and/or $t=1$. In particular, $\psi^{\prime}(t)=O\left(t^{p}\right)$ as $t \rightarrow 0+$ and $\psi^{\prime}(t)=O\left((1-t)^{q}\right)$ as $t \rightarrow 1-$, and $p$ and/or $q$ are not necessarily integers. Specifically, $\psi \in \widetilde{\mathcal{S}}_{p, q}$, where $\widetilde{\mathcal{S}}_{p, q}$ is a new class of variable transformations that is analogous to, and also contains, the class $\mathcal{S}_{p, q}$ due to the author 15.

(ii) More importantly, by adjusting the singular behavior of $\psi(t)$ at $t=0$ and $t=1$, that is, by choosing $p$ and $q$ suitably, the accuracy of the transformed rule $\widehat{G}_{n}[f]$ is optimized when $f(x)$ has algebraic endpoint singularities.

This approach to numerical integration was suggested by the author in the context of the trapezoidal rule in several publications. See [12, 13, 15], for example. We employ it in the context of Gauss-Legendre quadrature here, the variable transformations we use being from $\widetilde{\mathcal{S}}_{p, q}$.

Before proceeding further, we would like to present the line of thought that motivates the search for the special values of $p$ and $q$ that optimize the accuracy of $\widehat{G}_{n}[f]$. For this, we introduce the concept of quality of the numerical quadrature formula $\widehat{G}_{n}[f]$ in a way that is relevant to nonsymmetric transformations of the kind described in the preceding paragraph. This concept was first introduced in 12 for symmetric variable transformations and later extended to nonsymmetric ones in [13] and 15. In the present context, it is defined as follows: If $\psi^{\prime}(t) \sim \alpha t^{p}$ as $t \rightarrow 0+$ and $\psi^{\prime}(t) \sim \beta(1-t)^{q}$ as $t \rightarrow 1-$, and if $\widehat{E}_{n}[f]=I[f]-\widehat{G}_{n}[f]=O\left(n^{-\sigma}\right)$ as $n \rightarrow \infty$, for some $\sigma>0$, the quality of $\widehat{G}_{n}[f]$ is the ratio $\sigma / w$, where $w=\max \{p+1, q+1\}$. Note that the effective abscissas for $\widehat{G}_{n}[f]$ in (1.6) are $\xi_{n i} \equiv \psi\left(x_{n i}\right)$, and these cluster near $x=0$ and $x=1$ in the variable $x$ because, for all finite $i, \lim _{n \rightarrow \infty} x_{n i}=0$ and $\lim _{n \rightarrow \infty} x_{n-i+1}=1$, and hence $\lim _{n \rightarrow \infty} \psi\left(x_{n i}\right)=0$ and $\lim _{n \rightarrow \infty} \psi\left(x_{n-i+1}\right)=1$. In addition, the clustering increases with increasing $p$ and $q$, simultaneously with the accuracy of $\widehat{G}_{n}[f]$. Because too much clustering can cause overflows and underflows in floating-point arithmetic, hence is not desirable, we would like to get as much accuracy as possible from a given amount of clustering. In other words, we would like the quality of $\widehat{G}_{n}[f]$ to be as high as possible. This is achieved by the variable transformations in $\widetilde{\mathcal{S}}_{p, q}$ with special (not necessarily integer) values of $p$ and $q$. 
In the next section, we define the class $\widetilde{\mathcal{S}}_{p, q}$, and show how variable transformations $\psi(t)$ in this class can be constructed. We also study a member of this class that forms an extension of the Korobov [6] transformation, and show how it can be computed efficiently. In Section 3, we review a recent result of the author [14] concerning the asymptotic expansion of $E_{n}[u]$ when $u(x)$ is a function in $C^{\infty}(0,1)$ but has arbitrary algebraic singularities at $x=0$ and/or $x=1$. In Section 4 , we analyze the asymptotic behavior of $\widehat{E}_{n}[f]$ as $n \rightarrow \infty$ for the class of functions discussed in Section 3 and with variable transformations from $\widetilde{\mathcal{S}}_{p, q}$. We show that optimal results of remarkably high accuracies from Gauss-Legendre quadrature can be obtained by adjusting the singular behavior of the transformation $\psi(t)$ at $t=0$ and $t=1 \mathrm{in}$ a simple way. The main results of this section are Theorem 4.1 and Corollary 4.2, and the optimal results are the subject of parts (ii) of this theorem and corollary. Finally, in Section 5, we provide numerical examples that confirm the theory presented in Section 4 .

By now, it is clear that here we are dealing with variable transformations $\psi(t)$ that have power-like behavior at $t=0$ and $t=1$. Before we end this section, we would like to comment on the use of exponential type variable transformations $\psi(t)$, such as the tanh transformation of Sag and Szekeres [10], the IMT transformation of Iri, Moriguti, and Takasawa [3], and the double exponential transformation of Mori [9]. These transformations have the property that $\psi^{(i)}(0)=\psi^{(i)}(1)=0$, $i=1,2, \ldots$, and the transformed integrand $\widehat{f}(t)=f(\psi(t)) \psi^{\prime}(t)$, even when it has algebraic singularities at $x=0$ and/or $x=1$, satisfies $\widehat{f}^{(i)}(0)=\widehat{f}^{(i)}(1)=0, i=$ $0,1, \ldots$, which also means that $\widehat{f} \in C^{\infty}[0,1]$. Consequently, when Gauss-Legendre quadrature is applied to $\int_{0}^{1} \widehat{f}(t) d t$ as in (1.6), the error $\widehat{E}_{n}[f]$ in (1.7) satisfies $\widehat{E}_{n}[f]=O\left(n^{-\mu}\right)$ as $n \rightarrow \infty$, for every $\mu>0$. However, in floating point arithmetic, we may run into serious numerical problems in the form of underflows and overflows when using exponential type variable transformations, without achieving machine accuracy. This can be understood as follows: To begin with, the Gauss-Legendre abscissas $x_{n i}$ cluster near $x=0$ and $x=1$. Consequently, the clustering of the effective abscissas $\xi_{n i}=\psi\left(x_{n i}\right)$ in (1.6) near the endpoints $t=0$ and $t=1$ is much worse than those of the $x_{n i}$, because $\psi(t) \rightarrow 0$ as $t \rightarrow 0+$ and $\psi(t) \rightarrow 1$ as $t \rightarrow 1$ - exponentially. For this reason, when $f(0)$ and/or $f(1)$ are infinite, the floating-point computation of $f\left(\psi\left(x_{n i}\right)\right)$ for $i$ close to 1 and/or $n$ may result in overflows, even though $\widehat{f}(0)=\widehat{f}(1)=0$. In addition, for $i$ close to 1 and $n$, the corresponding $\psi^{\prime}\left(x_{n i}\right)$, which are part of of the weights in (1.6), become extremely small; consequently, their computation may result in underflows. See [1] and [7]. By comparison, for the variable transformations considered in the present work (and its predecessors as well), the problem of overflows and underflows is much milder.

\section{The Class $\widetilde{\mathcal{S}}_{p, q}$ And extended Korobov transformation}

In what follows we define the class $\widetilde{\mathcal{S}}_{p, q}$ and show how functions in this class

can be constructed. We also give the most immediate representative of $\widetilde{\mathcal{S}}_{p, q}$, the extended Korobov [6] transformation, which we call the $K^{p, q}$ transformation. 


\subsection{The class $\widetilde{\mathcal{S}}_{p, q}$.}

Definition 2.1. A function $\psi(t)$ is in the class $\widetilde{\mathcal{S}}_{p, q}$, with $p, q>0$ but arbitrary, if it has the following properties:

(1) $\psi \in C^{1}[0,1]$ and $\psi \in C^{\infty}(0,1) ; \psi(0)=0, \psi(1)=1$, and $\psi^{\prime}(t)>0$ on $(0,1)$.

(2) $\psi^{\prime}(t)$ has the following asymptotic expansions as $t \rightarrow 0+$ and $t \rightarrow 1-$ :

$$
\begin{aligned}
\psi^{\prime}(t) & \sim \sum_{i=0}^{\infty} \epsilon_{i}^{(0)} t^{p+i} \quad \text { as } t \rightarrow 0+; \quad \epsilon_{0}^{(0)}>0, \\
\psi^{\prime}(t) & \sim \sum_{i=0}^{\infty} \epsilon_{i}^{(1)}(1-t)^{q+i} \text { as } t \rightarrow 1-; \quad \epsilon_{0}^{(1)}>0 .
\end{aligned}
$$

Consequently,

$$
\begin{aligned}
& \psi(t) \sim \sum_{i=0}^{\infty} \epsilon_{i}^{(0)} \frac{t^{p+i+1}}{p+i+1} \quad \text { as } t \rightarrow 0+, \\
& \psi(t) \sim 1-\sum_{i=0}^{\infty} \epsilon_{i}^{(1)} \frac{(1-t)^{q+i+1}}{q+i+1} \quad \text { as } t \rightarrow 1-.
\end{aligned}
$$

(3) For each positive integer $k, \psi^{(k)}(t)$ has asymptotic expansions as $t \rightarrow 0+$ and $t \rightarrow 1$ - that are obtained by differentiating those of $\psi(t)$ term by term $k$ times.

Remark. When $p=q=m$ and $\psi^{\prime}(1-t)=\psi^{\prime}(t)$, which also implies that $\epsilon_{i}^{(0)}=\epsilon_{i}^{(1)}$, $i=0,1, \ldots$, in (2.1) and (2.2), $\psi(t)$ has the property that $\psi^{\prime}(t)$ is symmetric with respect to $t=1 / 2$ and $\psi(1-t)=1-\psi(t)$. We denote the class of these functions $\widetilde{\mathcal{S}}_{m}$. When $p=q=m$ but $\epsilon_{i}^{(0)} \neq \epsilon_{i}^{(1)}$ for some $i$ in (2.1) and (2.2), $\psi(t)$ does not have this symmetry property. This means that not all functions in the class $\widetilde{\mathcal{S}}_{m, m}$ have this symmetry property. Thus, $\widetilde{\mathcal{S}}_{m} \subset \widetilde{\mathcal{S}}_{m, m}$. Obviously, functions $\psi(t)$ in $\widetilde{\mathcal{S}}_{p, q}$ with $p \neq q$ do not have this symmetry property.

We now turn to the construction of functions in $\widetilde{\mathcal{S}}_{p, q}$. For this, we first define a class of functions we denote $\widetilde{\mathcal{K}}_{r}$ :

Definition 2.2. We say that a function $g(t)$ is in $\widetilde{\mathcal{K}}_{r}, r>0$, if

$$
g(t)=t^{r} \chi(t) ; \quad \chi \in C^{\infty}[0,1], \quad \chi(t)>0 \quad \text { on }[0,1],
$$

so that

$$
g(t)>0 \quad \text { on }(0,1] ; \quad g(t) \sim \sum_{i=0}^{\infty} \epsilon_{i} t^{r+i} \quad \text { as } t \rightarrow 0+.
$$

First, by invoking (2.1) and (2.2), it is easy to verify that $\psi \in \widetilde{\mathcal{S}}_{p, q}$ can be constructed as in the next theorem.

Theorem 2.3. Let $p>0$ and $q>0$, and define

$$
\psi(t)=\frac{\Theta(t)}{\Theta(1)} ; \quad \Theta(t)=\int_{0}^{t} v(u) w(1-u) d u ; \quad v \in \widetilde{\mathcal{K}}_{p}, \quad w \in \widetilde{\mathcal{K}}_{q} .
$$

Then $\psi \in \widetilde{\mathcal{S}}_{p, q}$. 
Next, we construct some special $\psi(t)$ in $\widetilde{\mathcal{S}}_{p, q}$. Now, if $\mu \in \widetilde{\mathcal{K}}_{1}, r>0$, and $g(t)=[\mu(t)]^{r}$, then $g \in \widetilde{\mathcal{K}}_{r}$. With this in mind, we choose

$$
v(t)=[\mu(t)]^{p}, \quad w(t)=[\mu(t)]^{q} ; \quad \mu \in \widetilde{\mathcal{K}}_{1}, \quad p, q>0 .
$$

With this choice of $v(t)$ and $w(t)$, (2.3) becomes

$$
\Theta(t)=\Theta_{p, q}(t)=\int_{0}^{t}[\mu(u)]^{p}[\mu(1-u)]^{q} d u .
$$

Then, the following facts are easy to verify:

$$
\begin{gathered}
\Theta_{p, q}(1)=\Theta_{q, p}(1), \\
\Theta_{p, q}(1)=\Theta_{p, q}(t)+\Theta_{q, p}(1-t)=\Theta_{q, p}(1), \\
\Theta_{p, q}(1)=\Theta_{p, q}(1 / 2)+\Theta_{q, p}(1 / 2)=\Theta_{q, p}(1) .
\end{gathered}
$$

Letting $\psi_{a, b}(t)=\Theta_{a, b}(t) / \Theta_{a, b}(1)$, from these, we also have

$$
\psi_{p, q}(t)=1-\psi_{q, p}(1-t) .
$$

In view of (2.8), it is sufficient to compute $\Theta_{p, q}(t)$ and $\Theta_{q, p}(t)$ for $t \in[0,1 / 2]$ in order to compute $\psi_{p, q}(t)$ for $t \in[0,1]$. Thus, after computing $\Theta_{p, q}(1)=\Theta_{q, p}(1)$ via (2.7), we have

$$
\psi_{p, q}(t)=\frac{\Theta_{p, q}(t)}{\Theta_{p, q}(1)}, \quad 0 \leq t \leq 1 / 2 ; \quad \psi_{p, q}(t)=1-\frac{\Theta_{q, p}(1-t)}{\Theta_{q, p}(1)}, \quad 1 / 2 \leq t \leq 1 .
$$

Clearly, with $\Theta_{p, q}(t)$ as in (2.4), when $p=q=m, \psi^{\prime}(t)$ is symmetric with respect to $t=1 / 2$, hence $\psi_{m, m} \in \widetilde{\mathcal{S}}_{m}$. Recall that $\widetilde{\mathcal{S}}_{m}$ is defined in the remark following Definition 2.1.

2.2. A representative of $\widetilde{\mathcal{S}}_{p, q}$ : The extended Korobov transformation. The simplest representative of the class $\widetilde{\mathcal{S}}_{p, q}$ seems to be an extension of the Korobov transformation, for which $\mu(t)=t$ in (2.4) $-(2.9)$. That is,

$$
\psi_{p, q}(t)=\frac{\Theta_{p, q}(t)}{\Theta_{p, q}(1)} ; \quad \Theta_{p, q}(t)=\int_{0}^{t} u^{p}(1-u)^{q} d u .
$$

We call it the $K^{p, q}$ transformation for short.

This transformation, with $p=q=m$ and integer $m$, was first proposed in Korobov [6]. It was generalized to $p \neq q$ precisely as in (2.10), again with integer $p$ and $q$, in Monegato and Scuderi 7 . We now propose to use (2.10) with arbitrary $p$ and $q$ that are not necessarily integers. Note also that, with $p=q=m$ in (2.10), the $K^{m, m}$ transformation is symmetric with respect to $t=1 / 2$, in the sense that $\psi_{m, m}^{\prime}(t)=\psi_{m, m}^{\prime}(1-t)$, hence is in $\widetilde{\mathcal{S}}_{m}$.

It is interesting to observe that $\psi_{p, q}(t)$, as given in (2.10), is actually related to the regularized incomplete Beta function $I_{x}(a, b)$ via

$$
\psi_{p, q}(t)=I_{t}(p+1, q+1) \text {. }
$$

Here

$$
I_{x}(a, b)=\frac{B_{x}(a, b)}{B(a, b)}
$$


where

$$
B(a, b)=\int_{0}^{1} \xi^{a-1}(1-\xi)^{b-1} d t, \quad B_{x}(a, b)=\int_{0}^{x} \xi^{a-1}(1-\xi)^{b-1} d t,
$$

and $B(a, b)$ and $B_{x}(a, b)$ are the Beta function and the incomplete Beta function, respectively. See Abramowitz and Stegun [1, p. 263, formulas 6.6.1 and 6.6.2].

When $p$ or $q$ is an integer, $\psi_{p, q}(t)$ has a finite expansion of the form $\psi(t)=$ $1-\sum_{i=0}^{p} \bar{\epsilon}_{i}^{(1)}(1-t)^{q+i+1}$ or $\psi(t)=\sum_{i=0}^{q} \bar{\epsilon}_{i}^{(0)} t^{p+i+1}$, respectively. Of course, $\psi_{p, q}(t)$ is a polynomial of degree $p+q+1$ when both $p$ and $q$ are integers.

In case $p$ or $q$ is an integer, $\psi_{p, q}(t)$ can also be computed by using the three-term recursion relations for the incomplete Beta function given in 1, p. 263, formulas 6.6.5-6.6.7], since initial conditions for these recursions are readily available, namely,

$$
\psi_{a, 0}(t)=t^{a+1} \quad \text { and } \quad \psi_{0, b}(t)=1-(1-t)^{b+1} .
$$

When $p$ and/or $q$ are not integers, $\psi_{p, q}(t)$ can be determined via (2.5)-(2.9) as follows:

(1) Compute $\Theta_{p, q}(1 / 2)$ and $\Theta_{q, p}(1 / 2)$, and set $c_{p, q}=c_{q, p}=\Theta_{p, q}(1 / 2)+$ $\Theta_{q, p}(1 / 2)$.

(2) For $t \in[0,1 / 2]$, compute $\Theta_{p, q}(t)$ and $\Theta_{q, p}(t)$, and set $\psi_{p, q}(t)=\Theta_{p, q}(t) / c_{p, q}$ and $\psi_{q, p}(t)=\Theta_{q, p}(t) / c_{q, p}$.

(3) For $t \in[1 / 2,1]$, compute $\psi_{p, q}(t)$ via $\psi_{p, q}(t)=1-\psi_{q, p}(1-t)$.

Clearly, before everything else, one must have a code for computing $\Theta_{a, b}(t)$ for $0 \leq t \leq 1 / 2$ to machine accuracy. This can be achieved by summing the infinite series representation

$$
\Theta_{a, b}(t)=\sum_{k=0}^{\infty}(-1)^{k}\left(\begin{array}{l}
b \\
k
\end{array}\right) \frac{t^{a+k+1}}{a+k+1}=\sum_{k=0}^{\infty} \frac{(-b)_{k}}{k !} \frac{t^{a+k+1}}{a+k+1},
$$

where $(z)_{k}=\prod_{i=1}^{k}(z+i-1)$ is the Pochhammer symbol. This series is obtained by integrating the expansion in powers of $u$ of $u^{a}(1-u)^{b}$ (about $u=0$ ) term by term. Its terms are ultimately of the same sign. It converges quickly for $t \in[0,1 / 2]$ since its $k$ th term tends to zero as $k \rightarrow \infty$ essentially like $k^{-b-2} t^{k}$ and hence, at worst, like $k^{-b-2} 2^{-k}$. Finally, the number of terms of the series required for computing its sum to machine accuracy becomes smaller as $t$ becomes smaller.

It is easy to see from (2.14) that $\Theta_{a, b}(t)$ can also be expressed in terms of the hypergeometric series as in

$$
\Theta_{a, b}(t)=\frac{t^{a+1}}{a+1}{ }_{2} F_{1}(-b, a+1 ; a+2 ; t) .
$$

2.3. The class $\mathcal{S}_{p, q}$ and the $\sin ^{p, q}$ transformation. As mentioned earlier, the class $\widetilde{\mathcal{S}}_{p, q}$ is analogous to the class $\mathcal{S}_{p, q}$ of the author [15. Actually, if $\psi \in \mathcal{S}_{p, q}$, then $\psi \in \widetilde{\mathcal{S}}_{p, q}$ as well, but with $\epsilon_{2 i+1}^{(0)}=0=\epsilon_{2 i+1}^{(1)}, i=0,1, \ldots$, in (2.1) and (2.2). Thus, $\mathcal{S}_{p, q}$ is a proper subset of $\widetilde{\mathcal{S}}_{p, q}$.

A representative of $\mathcal{S}_{p, q}$ is the $\sin ^{p, q}$ transformation that is defined via the integral representation

$$
\psi_{p, q}(t)=\frac{\Theta_{p, q}(t)}{\Theta_{p, q}(1)} ; \quad \Theta_{p, q}(t)=\int_{0}^{t}\left(\sin \frac{1}{2} \pi u\right)^{p}\left(\cos \frac{1}{2} \pi u\right)^{q} d u
$$


The case $p=q=m$, with integer $m$, was first given by the author in [11, and is known as the $\sin ^{m}$ transformation. The transformation in (2.16), again with integers $p$ and $q$, was proposed by Monegato and Scuderi in [7. The $\sin ^{m}$ transformation and the $\sin ^{p, q}$ transformations were extended to arbitrary $m, p$, and $q$ by the author in [12] and [15, respectively. Transformations in the class $\mathcal{S}_{p, q}$ are very effective (in fact, much more effective than other transformations in $\widetilde{\mathcal{S}}_{p, q}$ ) when used in conjunction with the trapezoidal rule approximation of the integrals in (1.1).

When $p, q$ and $m$ are integers, these transformations can be computed by short recurrences. For noninteger values of $p, q$ and $m$, their computation can be achieved with machine accuracy in an efficient manner by summing some infinite series representations of theirs. For details, see [12] and [15].

Finally, by making the substitution $\xi=\sin ^{2}\left(\frac{1}{2} \pi u\right)$ in the integral for $\Theta_{p, q}(t)$ in (2.16), we obtain the representation

$$
\Theta_{p, q}(t)=\frac{1}{\pi} \int_{0}^{S^{2}} \xi^{(p-1) / 2}(1-\xi)^{(q-1) / 2} d \xi ; \quad S=\sin \frac{\pi t}{2} .
$$

Now invoking (2.12) and (2.13), we realize that the $\sin ^{p, q}$ transformation in (2.16) is also related to the regularized Beta function $I_{x}(a, b)$ via

$$
\psi_{p, q}(t)=I_{S^{2}}\left(\frac{p+1}{2}, \frac{q+1}{2}\right) ; \quad S=\sin \frac{\pi t}{2} .
$$

\section{Asymptotic expansions for $E_{n}[u]$ In the PRESEnCE OF ALGEBRAIC ENDPOINT SINGULARITIES}

An important tool that we will be using in the sequel is the asymptotic expansion of $E_{n}[u]$ as $n \rightarrow \infty$ when the integrand $u(x)$ in $I[u]=\int_{0}^{1} u(x) d x$ has arbitrary algebraic singularities at the endpoints $x=0$ and/or $x=1$. The following result is Theorem 2.1 in [14]. It is a special case of a more general theorem in [14] in which the function $u(x)$ is allowed to have arbitrary algebraic-logarithmic singularities at the endpoints.

Theorem 3.1. Let $u \in C^{\infty}(0,1)$, and assume that $u(x)$ has the asymptotic expansions

$$
u(x) \sim \sum_{s=0}^{\infty} A_{s} x^{\alpha_{s}} \quad \text { as } x \rightarrow 0+; \quad u(x) \sim \sum_{s=0}^{\infty} B_{s}(1-x)^{\beta_{s}} \quad \text { as } x \rightarrow 1-,
$$

where $A_{s}$ and $B_{s}$ are some constants and $\alpha_{s}$ and $\beta_{s}$ are in general complex and satisfy

$$
\begin{aligned}
-1<\Re \alpha_{0} \leq \Re \alpha_{1} \leq \Re \alpha_{2} \leq \cdots ; & \lim _{s \rightarrow \infty} \Re \alpha_{s}=+\infty, \\
-1<\Re \beta_{0} \leq \Re \beta_{1} \leq \Re \beta_{2} \leq \cdots ; & \lim _{s \rightarrow \infty} \Re \beta_{s}=+\infty .
\end{aligned}
$$

Assume, furthermore, that for each $k=1,2, \ldots$, the $k$ th derivative of $u(x)$ also has asymptotic expansions as $x \rightarrow 0+$ and $x \rightarrow 1-$ that are obtained by differentiating those in (3.1) term by term. Then, with $h=(n+1 / 2)^{-2}$ and $\mathbb{Z}^{+}=\{0,1,2, \ldots\}$, there holds

$$
E_{n}[u] \sim \sum_{\substack{s=0 \\ \alpha_{s} \notin \mathbb{Z}^{+}}}^{\infty} \sum_{k=1}^{\infty} a_{s k} h^{\alpha_{s}+k}+\sum_{\substack{s=0 \\ \beta_{s} \notin \mathbb{Z}^{+}}}^{\infty} \sum_{k=1}^{\infty} b_{s k} h^{\beta_{s}+k} \quad \text { as } n \rightarrow \infty .
$$


Here, $a_{s k}$ and $b_{s k}$ are some constants independent of $n$. Those $a_{s k}$ and $b_{s k}$ corresponding to $\alpha_{s}, \beta_{s} \in \mathbb{Z}^{+}$are simply zero, hence absent from the summations in (3.3).

Remarks.

(1) If $u(x)=x^{\mu}(1-x)^{\nu} g(x), g(x)$ being infinitely differentiable on [0,1], then $u(x)$ satisfies the conditions of the theorem. In such a case, if $g(x)$ has full Taylor series at $x=0$ and $x=1$, we have $\gamma_{s}=\mu+s$ and $\delta_{s}=\nu+s$, $s=0,1, \ldots$. Note that this $u(x)$ has an algebraic branch singularity at $x=0$ if $\mu$ is not a nonnegative integer. Similarly, it has an algebraic branch singularity at $x=1$ if $\nu$ is not a nonnegative integer.

(2) It is clear from (3.3) that nonnegative integer powers $x^{j}$ and $(1-x)^{j}$, even when they are present in the asymptotic expansions of $u(x)$ given in (3.1), do not contribute to the asymptotic expansion of $E_{n}[u]$ in (3.3). Consequently, if $\alpha_{\mu}$ is the first of the $\alpha_{s}$ that is different from $0,1,2, \ldots$, and if $\beta_{\nu}$ is the first of the $\beta_{s}$ that is different from $0,1,2, \ldots$, then

$$
E_{n}[f]=O\left(h^{\sigma+1}\right) \quad \text { as } n \rightarrow \infty ; \quad \sigma=\min \left\{\Re \alpha_{\mu}, \Re \beta_{\nu}\right\} .
$$

This is a crucial observation that forms the motivation for the present paper: By using a variable transformation in $\widetilde{\mathcal{S}}_{p, q}$ first, and by choosing $p$ and $q$ in a suitable manner, we are able to cause several of the dominant powers of $t$ and $(1-t)$ in the asymptotic expansions, as $t \rightarrow 0+$ and as $t \rightarrow 1-$, of the transformed integrand $\widehat{f}(t)$ in (1.5) to be nonnegative integers; thus forcing the asymptotic expansion of $\widehat{E}_{n}[f]$ to start with a very high power of $h$.

\section{Analysis of $\widehat{E}_{n}[f]$ With Class $\widetilde{\mathcal{S}}_{p, q}$ TRAnsformations}

In this section, we analyze the behavior of $\widehat{E}_{n}[f]$, the error in $\widehat{G}_{n}[f]$ given in (1.6), as $n \rightarrow \infty$, when the integrand $f(x)$ is infinitely differentiable in $(0,1)$ and possibly has algebraic singularities at $x=0$ and/or $x=1$ of the type discussed in Theorem 3.1 .

Here is our main result:

Theorem 4.1. Let $f \in C^{\infty}(0,1)$, and assume that $f(x)$ has the asymptotic expansions

$$
f(x) \sim \sum_{s=0}^{\infty} c_{s} x^{\gamma_{s}} \quad \text { as } x \rightarrow 0+; \quad f(x) \sim \sum_{s=0}^{\infty} d_{s}(1-x)^{\delta_{s}} \quad \text { as } x \rightarrow 1-.
$$

Here $\gamma_{s}$ and $\delta_{s}$ are distinct complex numbers that satisfy

$$
\begin{aligned}
& -1<\Re \gamma_{0} \leq \Re \gamma_{1} \leq \Re \gamma_{2} \leq \cdots ; \quad \lim _{s \rightarrow \infty} \Re \gamma_{s}=+\infty, \\
& -1<\Re \delta_{0} \leq \Re \delta_{1} \leq \Re \delta_{2} \leq \cdots ; \quad \lim _{s \rightarrow \infty} \Re \delta_{s}=+\infty .
\end{aligned}
$$

Assume, furthermore, that for each positive integer $k, f^{(k)}(x)$ has asymptotic expansions as $x \rightarrow 0+$ and $x \rightarrow 1$ - that are obtained by differentiating those of $f(x)$ term by term $k$ times. Let $I[f]=\int_{0}^{1} f(x) d x$, and make the transformation of variable $x=\psi(t)$, where $\psi \in \widetilde{\mathcal{S}}_{p, q}$, in $I[f]$. Finally, approximate $I[f]$ via the n-point GaussLegendre rule $\widehat{G}_{n}[f]=\sum_{i=1}^{n} w_{n i} f\left(\psi\left(x_{n i}\right)\right) \psi^{\prime}\left(x_{n i}\right)$. Then, with $h=(n+1 / 2)^{-2}$ and 
$\mathbb{Z}^{+}=\{0,1,2, \ldots\}, \widehat{E}_{n}[f]$ has an asymptotic expansion of the form

$$
\begin{gathered}
\widehat{E}_{n}[f] \sim \sum_{\substack{s=0 \\
\gamma_{s}(p+1)+p \notin \mathbb{Z}^{+}}}^{\infty} \sum_{j=0}^{\infty} a_{s j} h^{\left(\gamma_{s}+1\right)(p+1)+j} \\
+\sum_{\substack{s=0 \\
\delta_{s}(q+1)+q \notin \mathbb{Z}^{+}}}^{\infty} \sum_{j=0}^{\infty} b_{s j} h^{\left(\delta_{s}+1\right)(q+1)+j} \\
\text { as } n \rightarrow \infty .
\end{gathered}
$$

As a result, the following hold:

(i) For arbitrary $p$ and $q$, in the worst case,

(4.4) $\widehat{E}_{n}[f]=O\left(h^{\omega}\right) \quad$ as $n \rightarrow \infty ; \quad \omega=\min \left\{\left(\Re \gamma_{0}+1\right)(p+1),\left(\Re \delta_{0}+1\right)(q+1)\right\}$.

(ii) If $\gamma_{0}$ and $\delta_{0}$ are real, and

$$
p=\left(k-\gamma_{0}\right) /\left(\gamma_{0}+1\right)>0, \quad q=\left(l-\delta_{0}\right) /\left(\delta_{0}+1\right)>0, \quad k, l \in \mathbb{Z}^{+},
$$

then, in the worst case, the outer summations in the asymptotic expansion in (4.3) begin with $s=1$ terms, and we have

$\widehat{E}_{n}[f]=O\left(h^{\omega}\right) \quad$ as $n \rightarrow \infty ; \quad \omega=\min \left\{\left(\Re \gamma_{1}+1\right)(p+1),\left(\Re \delta_{1}+1\right)(q+1)\right\}$.

A more refined version of this result is

$\widehat{E}_{n}[f]=O\left(h^{\omega}\right) \quad$ as $n \rightarrow \infty ; \quad \omega=\min \left\{\left(\Re \gamma_{\mu}+1\right)(p+1),\left(\Re \delta_{\nu}+1\right)(q+1)\right\}$,

where $\gamma_{\mu}$ is the first of the $\gamma_{s}, s \geq 1$, for which $\left(\gamma_{s}+1\right)(p+1) \neq$ integer, and $\delta_{\nu}$ is the first of the $\delta_{s}, s \geq 1$, for which $\left(\delta_{s}+1\right)(q+1) \neq$ integer.

Remark. The special values of the parameters $p$ and $q$ given in (4.5) in part (ii) of the theorem are the optimal values we alluded to earlier in this work. Clearly, for these values of $p$ and $q, \widehat{E}_{n}[f]$ is much better than the ones achieved by other values.

Proof. It is clear that Theorem 3.1 applies because the transformed integrand $\widehat{f}(t)=f(\psi(t)) \psi^{\prime}(t)$ is as described there. Thus, we need to analyze the asymptotic expansions of $\widehat{f}(t)$ as $t \rightarrow 0+$ and $t \rightarrow 1-$. To proceed with this analysis, we need the following: Let $v(\xi)$ denote generically any function that has an asymptotic expansion of the form $\sum_{i=0}^{\infty} v_{i} \xi^{i}$ as $\xi \rightarrow 0+$. Then

$$
g(\xi)=\xi^{r} v(\xi) \quad \Rightarrow \quad[g(\xi)]^{\mu}=\xi^{\mu r} v(\xi)
$$

and

$$
g_{i}(\xi)=\xi^{r_{i}} v(\xi), \quad i=1, \ldots, k, \quad \Rightarrow \quad \prod_{i=1}^{k} g_{i}(\xi)=\xi^{r} v(\xi), \quad r=\sum_{i=1}^{k} r_{i} .
$$

Because $\psi(t) \rightarrow 0$ as $t \rightarrow 0+$ and $\psi(t) \rightarrow 1$ as $t \rightarrow 1-$, from (4.1) and (4.2), we first have

$$
\begin{aligned}
& \widehat{f}(t) \sim \sum_{s=0}^{\infty} c_{s}[\psi(t)]^{\gamma_{s}} \psi^{\prime}(t) \quad \text { as } t \rightarrow 0+; \\
& \widehat{f}(t) \sim \sum_{s=0}^{\infty} d_{s}[1-\psi(t)]^{\delta_{s}} \psi^{\prime}(t) \quad \text { as } t \rightarrow 1-.
\end{aligned}
$$


Invoking (2.1) and (2.2), and re-expanding these asymptotic series, we have that the $s$ th term in the first of these series contributes the sum

$$
\begin{aligned}
K_{s}^{(0)}(t) & :=\sum_{i=0}^{\infty} e_{s i}^{(0)} t^{\gamma_{s}(p+1)+p+i} \quad \text { as } t \rightarrow 0+; \\
e_{s 0}^{(0)} & =c_{s}\left[\epsilon_{0}^{(0)}\right]^{\gamma_{s}+1} /(p+1)^{\gamma_{s}} \neq 0,
\end{aligned}
$$

whereas the $s$ th term in the second series contributes the sum

$$
\begin{aligned}
K_{s}^{(1)}(t) & :=\sum_{i=0}^{\infty} e_{s i}^{(1)}(1-t)^{\delta_{s}(q+1)+q+i} \quad \text { as } t \rightarrow 1-; \\
e_{s 0}^{(1)} & =d_{s}\left[\epsilon_{0}^{(1)}\right]^{\delta_{s}+1} /(q+1)^{\delta_{s}} \neq 0 .
\end{aligned}
$$

Substituting (4.9) and (4.10) in (4.8), and applying Theorem 3.1, we first obtain

$$
\begin{aligned}
& \widehat{E}_{n}[f] \sim \sum_{s=0}^{\infty} \sum_{i=0}^{\infty} \sum_{k=1}^{\infty} C_{s i k}^{(0)} h^{\gamma_{s}(p+1)+p+i+k} \\
& +\sum_{s=0}^{\infty} \sum_{i=0}^{\infty} \sum_{k=1}^{\infty} C_{s i k}^{(1)} h^{\delta_{s}(q+1)+q+i+k} \quad \text { as } n \rightarrow \infty .
\end{aligned}
$$

Since $i+k=1,2,3, \ldots$ in both summations in (4.11), the summations over $i$ and $k$ can be combined into one, and this results in (4.3).

Thus, by Theorem [3.1, the most dominant terms in the expansion of $\widehat{E}_{n}[f]$ as $n \rightarrow \infty$ are $e_{00}^{(0)} h^{\left(\gamma_{0}+1\right)(p+1)}$ coming from the endpoint $x=0$, and $e_{00}^{(1)} h^{\left(\delta_{0}+1\right)(q+1)}$ coming from the endpoint $x=1$. This proves (4.4).

To prove (4.6), we note from Theorem 3.1 that if we choose $p$ such that $\gamma_{s}(p+1)+p$ is a nonnegative integer, then all the powers of $t$ in the asymptotic expansion $K_{s}^{(0)}(t)$ of (4.9) are also nonnegative integers, hence do not contribute to the asymptotic expansion of $\widehat{E}_{n}[f]$. Similarly, if we choose $q$ such that $\delta_{s}(q+1)+q$ is a nonnegative integer, then all the powers of $(1-t)$ in the asymptotic expansion $K_{s}^{(1)}(t)$ of (4.10) are also nonnegative integers, hence do not contribute to the asymptotic expansion of $\widehat{E}_{n}[f]$. Thus, when $\gamma_{0}$ and $\delta_{0}$ are real, if we choose $p>0$ and $q>0$ such that $\gamma_{0}(p+1)+p=k$ and $\delta_{0}(q+1)+q=l$, where $k$ and $l$ are nonnegative integers, then neither $K_{0}^{(0)}(t)$ in (4.9) nor $K_{0}^{(1)}(t)$ in (4.10) contributes to the asymptotic expansion of $\widehat{E}_{n}[f]$. By (3.4), the largest terms that possibly contribute are (i) $e_{10}^{(0)} t^{\gamma_{1}(p+1)+p}$, the first term of $K_{1}^{(0)}(t)$, provided $\gamma_{1}(p+1)+p$ is not a nonnegative integer, and (ii) $e_{10}^{(1)}(1-t)^{\delta_{1}(q+1)+q}$, the first term of $K_{1}^{(1)}(t)$, provided $\delta_{1}(q+1)+q$ is not a nonnegative integer. Under these conditions, the contributions of these terms to $\widehat{E}_{n}[f]$ are $a^{(0)} h^{\left(\gamma_{1}+1\right)(p+1)}$ and $a^{(1)} h^{\left(\delta_{1}+1\right)(q+1)}$, respectively, $a^{(0)}, a^{(1)}$ being some constants. This completes the proof of (4.6). The proof of (4.7) is the same.

Corollary 4.2. In case $f(x)=x^{\mu}(1-x)^{\nu} g(x)$ and $g \in C^{\infty}[0,1]$, such that $\Re \mu>$ $-1, \Re \nu>-1$ but $\mu \notin \mathbb{Z}^{+}, \nu \notin \mathbb{Z}^{+}$, the following hold:

(i) In the worst case,

$$
\widehat{E}_{n}[f]=O\left(h^{\omega}\right) \quad \text { as } n \rightarrow \infty ; \quad \omega=\min \{(\Re \mu+1)(p+1),(\Re \nu+1)(q+1)\} .
$$


(ii) If $\mu$ and $\nu$ are real, and if

$$
p=(k-\mu) /(\mu+1)>0, \quad q=(l-\nu) /(\nu+1)>0, \quad k, l \in \mathbb{Z}^{+},
$$

then, in the worst case,

$$
\widehat{E}_{n}[f]=O\left(h^{\omega}\right) \quad \text { as } n \rightarrow \infty ; \quad \omega=\min \{(\mu+2)(p+1),(\nu+2)(q+1)\} .
$$

Remark. The special values of the parameters $p$ and $q$ given in (4.13) in part (ii) of the corollary are the optimal values.

When $\mu=\nu=\lambda$ in part (ii) of Corollary 4.2 we can use a class $\widetilde{\mathcal{S}}_{m, m}$ variable transformation with $m=(k-\lambda) /(\lambda+1)$ to obtain the optimal result $\widehat{E}_{n}[f]=O\left(h^{\omega}\right)$ as $h \rightarrow 0$, where $\omega=(\lambda+2)(m+1)$.

When $\mu \neq \nu$ in part (ii) of Corollary 4.2, we choose the integers $k$ and $l$ such that $(\mu+2)(p+1) \approx(\nu+2)(q+1)$, that is,

$$
\frac{k+1}{l+1} \approx \frac{\nu+2}{\nu+1} \cdot \frac{\mu+1}{\mu+2} .
$$

(Thus, by choosing $k$ first, we can determine $l$, and vice versa.) This guarantees that the singularities of the transformed integrand $\widehat{f}(t)=f(\psi(t)) \psi^{\prime}(t)$ at the endpoints are of approximately the same strength.

Note that, when $f(x)$ is as in Corollary $4.2, I[f]$ can be computed by GaussJacobi quadrature formulas for the weight function $w(x)=x^{\mu}(1-x)^{\nu}$. The computation of the abscissas and weights for these formulas with arbitrary values of $\mu$ and $\nu$ is possible, provided one has suitable software. No special software is needed for computing $\widehat{G}_{n}[f]$ since the abscissas and weights for Gauss-Legendre quadrature formulas have been tabulated extensively, hence are readily available, and the $K^{p, q}$ transformation can be computed to machine accuracy in an efficient manner, as explained in subsection 2.2. This makes the use of the combined numerical quadrature rule $\widehat{G}_{n}[f]$ quite simple and accessible. In addition, by choosing $p$ and $q$ optimally, we are able to attain very high accuracies with a small number of function evaluations, as mentioned earlier and as will be illustrated in the next section. These facts make the combined optimal numerical quadrature rule competitive.

In Corollary 4.2, if the values of the function $g(x)$ at the endpoints are known, then even better results can be obtained as stated in the next corollary.

Corollary 4.3. Let $\bar{f}(x)=x^{\mu}(1-x)^{\nu} \bar{g}(x)$ and $\bar{g} \in C^{\infty}[0,1]$, such that $\Re \mu>-1$, $\Re \nu>-1$ but $\mu \notin \mathbb{Z}^{+}, \nu \notin \mathbb{Z}^{+}$. Assume that $\bar{g}(0)$ and $\bar{g}(1)$ are known and that $|\bar{g}(0)|+|\bar{g}(1)| \neq 0$. Let $U(x)$ be the linear interpolant to $\bar{g}(x)$ at $x=0$ and $x=1$, that is, $U(x)=\bar{g}(0)+[\bar{g}(1)-\bar{g}(0)] x$. Also, let $\bar{f}_{0}(x)=x^{\mu}(1-x)^{\nu} U(x)$ and $f(x)=\bar{f}(x)-\bar{f}_{0}(x)$. Take $\mathcal{G}_{n}[\bar{f}]=\widehat{G}_{n}[f]+I\left[\bar{f}_{0}\right]$ as the approximation to $I[\bar{f}]$, and denote $\mathcal{E}_{n}[\bar{f}]=I[\bar{f}]-\mathcal{G}_{n}[\bar{f}]$. Then the following hold:

(i) In the worst case,

$\mathcal{E}_{n}[\bar{f}]=O\left(h^{\omega}\right) \quad$ as $n \rightarrow \infty ; \quad \omega=\min \{(\Re \mu+2)(p+1),(\Re \nu+2)(q+1)\}$.

(ii) If $\mu$ and $\nu$ are real, and if

$$
p=(k-\mu-1) /(\mu+2)>0, \quad q=(l-\nu-1) /(\nu+2)>0, \quad k, l \in \mathbb{Z}^{+},
$$

then, in the worst case,

$$
\mathcal{E}_{n}[\bar{f}]=O\left(h^{\omega}\right) \quad \text { as } n \rightarrow \infty ; \quad \omega=\min \{(\mu+3)(p+1),(\nu+3)(q+1)\} .
$$


Here,

$$
I\left[\bar{f}_{0}\right]=\bar{g}(0) B(\mu+1, \nu+1)+[\bar{g}(1)-\bar{g}(0)] B(\mu+2, \nu+1),
$$

where $B(a, b)$ is the Beta function defined in (2.13).

Proof. First, $I[\bar{f}]=I[f]+I\left[\bar{f}_{0}\right]$. Therefore, $\mathcal{E}_{n}[\bar{f}]=\widehat{E}_{n}[f]$. Next,

$$
f(x)=x^{\mu}(1-x)^{\nu} v(x), \quad v(x)=\bar{g}(x)-U(x) \text { and } v(0)=v(1)=0 .
$$

Consequently, $f(x)$ is of the form $f(x)=x^{\mu+1}(1-x)^{\nu+1} g(x)$ for some $g \in C^{\infty}[0,1]$. Now applying Corollary 4.2 with $f(x)$, the result follows.

\section{Numerical EXAmples}

In this section, we provide three examples to illustrate the validity of the results of the preceding section. Even though the combined computational technique that we use is the same for all three examples, each example brings something special with respect to the asymptotics of $\widehat{E}_{n}[f]$.

The computations for these examples were done in quadruple-precision arithmetic (approximately 35 decimal digits) using both the $K^{p, q}$ (extended Korobov) transformation and the $\sin ^{p, q}$ transformation. Since the numerical results with both transformations are very similar (recall that they both belong to $\widetilde{S}_{p, q}$ ), we show only the ones obtained with the $K^{p, q}$ transformation.

Now, with quadruple-precision arithmetic, we need the abscissas $x_{n i}$ and the weights $w_{n i}$ to have machine precision (about 35 decimal digits). In the absence of software that can produce them with this accuracy, we can use the numerical approach mentioned in [2, pp. 113-114] to achieve this goal.

Note that in our computations we have taken $n=2^{k}$. Thus, if $\widehat{E}_{n}[f] \sim C h^{\omega}$ as $n \rightarrow \infty$ for some constant $C$, then the numbers

$$
\rho_{p, q, k}=\frac{1}{\log 2} \cdot \log \left(\frac{\left|\widehat{E}_{2^{k}}[f]\right|}{\left|\widehat{E}_{2^{k+1}}[f]\right|}\right)
$$

tend to $2 \omega$ as $n \rightarrow \infty$, because $h \sim n^{-2}$ as $n \rightarrow \infty$. In our examples, we provide these numbers as a confirmation of our theory of convergence.

In Section 1, we described the concept of the quality of a numerical quadrature formula in the context of the rules $\widehat{G}_{n}[f]$. There, we concluded that it is important that the quality of $\widehat{G}_{n}[f]$ be as high as possible. As seen from parts (ii) of Theorem 4.1 and Corollary 4.2, this is achieved by the variable transformations in $\widetilde{\mathcal{S}}_{p, q}$ with special (not necessarily integer) values of $p$ and $q$ in (4.5) and (4.13). Indeed, comparing the $\omega$ in (4.4) with those in (4.6) and (4.7), for example, we realize that the quadrature rule $\widehat{G}_{n}[f]$ with optimal $p$ and $q$ has substantially higher quality than that with $p$ and $q$ that are even slightly different from the optimal ones. This is precisely how we compare the effect of optimal and nonoptimal choices of $p$ and $q$ in our examples.

Example 5.1. Consider the integral

$$
\int_{0}^{1} x^{\mu} d x=\frac{1}{1+\mu}, \quad \mu>-1
$$

In this case, we have

$$
f(x)=x^{\mu} \quad \text { and } \quad f(x)=\sum_{s=0}^{\infty}(-1)^{s}\left(\begin{array}{c}
\mu \\
s
\end{array}\right)(1-x)^{s} .
$$


Of these, the first is a single-term series representing $f(x)$ asymptotically as $x \rightarrow 0+$ with $\gamma_{0}=\mu$, while the second is a (convergent) series representing $f(x)$ asymptotically as $x \rightarrow 1-$ with $\delta_{s}=s, s=0,1, \ldots$. (Note that now, in the notation of Corollary 4.2, $\nu=0$.) Thus, if we choose $p$ and $q$ arbitrarily, we will obtain, by part (i) of Theorem 4.1 and Corollary 4.2 ,

$$
\widehat{E}_{n}[f]=O\left(h^{\omega}\right) \quad \text { as } n \rightarrow \infty ; \quad \omega=\min \{(\mu+1)(p+1),(q+1)\} .
$$

In case $p=(k-\mu) /(1+\mu)$ and $q=l$, with $k$ and $l$ nonnegative integers, we will obtain, by part (ii) of Theorem 4.1

$$
\widehat{E}_{n}[f]=O\left(h^{\omega}\right) \quad \text { as } n \rightarrow \infty, \quad \text { for every } \omega>0 .
$$

This is so because the asymptotic expansion of $\widehat{E}_{n}[f]$ is empty due to the fact that the asymptotic expansion of $f(x)$ as $x \rightarrow 0+$ consists of only the term $x^{\mu}$. In the special case with $k=0$ and $l=0$, we have $p=-\mu /(\mu+1)$ and $q=0$, so that the extended Korobov transformation becomes simply $\psi_{p, q}(t)=t^{1 /(\mu+1)}$ and the transformed integrand becomes $\widehat{f}(t)=1 /(\mu+1)$, which implies that $\widehat{E}_{n}[f]=0$ for every $n$ with this $\psi_{p, q}(t)$.

In our computations, we have taken $\mu=0.1$

In Tables 1 and 3 we give the errors $\left|\widehat{E}_{n}[f]\right|$ for $n=2^{k}, k=1, \ldots, 6$, obtained with the $K^{p, q}$ transformation. In column $j$ of Table 1, we have chosen (nonoptimally) $p=(j-\mu) /(1+\mu)+0.1$ and $q=j+0.1$. In column $j$ of Table 3. we have chosen $p=(j-\mu) /(1+\mu)$ and $q=j$, which are the optimal values of $p$ and $q$. The superior convergence of $\widehat{G}_{n}[f]$ with optimal $p$ and $q$ is clearly demonstrated in Table 3 ,

In Table 2, we give the numbers $\rho_{p, q, k}$ for those values of $p$ and $q$ that are used in Table 1 It is seen that, with increasing $k$, the $\rho_{p, q, k}$ are tending to $2(\mu+1)(p+1)$, completely in accordance with Theorem 4.1 and Corollary 4.2 .

Example 5.2. Consider the integral

$$
\int_{0}^{1} f(x)=\pi 2^{1 / 4}, \quad f(x)=\frac{x^{-3 / 4}(1-x)^{-1 / 4}}{1+x} .
$$

Thus, $f(x)=x^{\mu}(1-x)^{\nu} g(x)$, precisely as in Corollary 4.2, with

$$
\mu=-3 / 4, \quad \nu=-1 / 4, \quad g(x)=1 /(1+x) .
$$

Therefore, $\gamma_{0}=\mu$ and $\delta_{0}=\nu$ in Theorem 4.1. Thus, if we choose $p$ and $q$ arbitrarily, we will obtain, by part (i) of Theorem 4.1 and Corollary 4.2 .

$$
\widehat{E}_{n}[f]=O\left(h^{\omega}\right) \quad \text { as } n \rightarrow \infty ; \quad \omega=\min \{(p+1) / 4,3(q+1) / 4\} .
$$

In case $p=(k-\mu) /(1+\mu)=4 k+3$ and $q=(l-\nu) /(1+\nu)=(4 l+1) / 3$, with $k$ and $l$ nonnegative integers, we will obtain, by part (ii) of Theorem 4.1 and Corollary 4.2 .

$$
\widehat{E}_{n}[f]=O\left(h^{\omega}\right) \quad \text { as } n \rightarrow \infty ; \quad \omega=(\nu+2)(q+1)=7(l+1) / 3,
$$

because now $(\mu+s)(p+1)+p$ are all nonnegative integers for all $s=0,1, \ldots$.

In Tables 4 and 6] we give the errors $\left|\widehat{E}_{n}[f]\right|$ for $n=2^{k}, k=1, \ldots, 6$, obtained with the $K^{p, q}$ transformation. In column $j$ of Table 4, we have chosen (nonoptimally) $p=4 j+3+0.1$ and $q=(4 j+3) / 3+0.1$, whereas in column $j$ of Table 6 , we have chosen $p=4 j+3$ and $q=(4 j+3) / 3$, which are optimal values of $p$ and $q$. It is interesting to note that, with $j+1=3,6,9, \ldots$, we have that $(\nu+s)(q+1)+q$, 
$s=0,1, \ldots$, are nonnegative integers; therefore, the asymptotic expansion of $\widehat{E}_{n}[f]$ is now empty, that is,

$$
\widehat{E}_{n}[f]=O\left(h^{\omega}\right) \quad \text { as } n \rightarrow \infty, \quad \text { for every } \omega>0 .
$$

This explains the very high quality of the results in the $j=2$ column in Table 6 .

In Tables 5 and 7 , we give the numbers $\rho_{p, q, k}$ that result from Tables 4 and 6 , respectively. It is seen that, with increasing $k$, the $\rho_{p, q, k}$ are tending to $2 \omega$.

Example 5.3. Consider the integral

$$
\int_{0}^{1} f(x)=0, \quad f(x)=\frac{d}{d x}\left[x^{\mu+1}(1-x)^{\nu+1} w(x)\right], \quad \mu, \nu>-1, \quad w \in C^{\infty}[0,1] .
$$

In this case, we have

$$
f(x)=x^{\mu}(1-x)^{\nu} g(x),
$$

where

$$
g(x)=[(\mu+1)(1-x)-(\nu+1) x] w(x)+x(1-x) w^{\prime}(x) .
$$

If $w(0)$ and $w(1)$ are both nonzero, we have that $g(0)$ and $g(1)$ are both nonzero as well, and this implies that $\gamma_{0}=\mu$ and $\delta_{0}=\nu$. Thus, if we choose $p$ and $q$ arbitrarily, we will obtain, by part (i) of Theorem 4.1 and Corollary 4.2 ,

$$
\widehat{E}_{n}[f]=O\left(h^{\omega}\right) \quad \text { as } n \rightarrow \infty ; \quad \omega=\min \{(\mu+1)(p+1),(\nu+1)(q+1)\} .
$$

If $p=(k-\mu) /(1+\mu)$ and $q=(l-\nu) /(1+\nu)$, with $k$ and $l$ nonnegative integers, we will obtain, by part (ii) of Theorem 4.1 and Corollary 4.2.

$$
\widehat{E}_{n}[f]=O\left(h^{\omega}\right) \quad \text { as } n \rightarrow \infty ; \quad \omega=\min \{(\mu+2)(p+1),(\nu+2)(q+1)\} .
$$

In our computations, we have taken $\mu=1 / 4$ and $\nu=-1 / 3$ and $w(x)=1 /(1+x)$.

In Tables 8 and 11, we give the errors $\left|\widehat{E}_{n}[f]\right|$ for $n=2^{k}, k=1, \ldots, 6$, obtained with the $K^{p, q}$ transformation. In column $j$ of Table 8 , we have chosen (nonoptimally) $p=(4 j-1) / 5+0.1$ and $q=(3 j+1) / 2+0.1$, whereas in column $j$ of Table 10. we have chosen $p=(4 j-1) / 5$ and $q=(3 j+1) / 2$, which are optimal values of $p$ and $q$. Note that, with $j+1=5,10,15, \ldots$, we have that $(\mu+s)(p+1)+p$, $s=0,1, \ldots$ are nonnegative integers. Similarly, with $j+1=2,4,6, \ldots$, we have that $(\nu+s)(q+1)+q, s=0,1, \ldots$ are nonnegative integers. Clearly, this causes an infinite number of terms in the asymptotic expansion of $\widehat{E}_{n}[f]$ to disappear. Thus, when we look for the precise value of $\omega$ in Corollary 4.2. we need to take these vanishing terms into account.

In Tables 9 and 11, we give the numbers $\rho_{p, q, k}$ that result from Tables 8 and 10. respectively. It is seen that, with increasing $k$, the $\rho_{p, q, k}$ are tending to $2 \omega$, completely in accordance with Theorem 4.1 and Corollary 4.2 , 
TABLE 1. Errors $\widehat{E}_{n}[f]$ for the integral of Example 5.1 obtained with $n=2^{k}, k=1(1) 6$, and with the $K^{p, q}$ transformation. In column $j$, we have chosen $p=(j-\mu) /(1+\mu)+0.1$ and $q=j+0.1$. (Nonoptimal $p, q$.)

\begin{tabular}{||r||c|c|c|c|c||}
\hline$n$ & $j=0$ & $j=1$ & $j=2$ & $j=3$ & $j=4$ \\
\hline \hline 2 & $1.67 D-02$ & $1.41 D-02$ & $1.72 D-01$ & $3.39 D-01$ & $4.82 D-01$ \\
4 & $4.40 D-03$ & $6.77 D-04$ & $3.29 D-04$ & $1.01 D-03$ & $1.57 D-02$ \\
8 & $1.07 D-03$ & $4.18 D-05$ & $4.56 D-06$ & $1.06 D-06$ & $6.82 D-07$ \\
16 & $2.46 D-04$ & $2.48 D-06$ & $6.67 D-08$ & $3.46 D-09$ & $3.01 D-10$ \\
32 & $5.49 D-05$ & $1.42 D-07$ & $9.64 D-10$ & $1.24 D-11$ & $2.62 D-13$ \\
64 & $1.21 D-05$ & $7.90 D-09$ & $1.36 D-11$ & $4.39 D-14$ & $2.31 D-16$ \\
\hline
\end{tabular}

TABLE 2. The numbers $\rho_{p, q, k}=\frac{1}{\log 2} \cdot \log \left(\frac{\left|\widehat{E}_{2^{k}}[f]\right|}{\left|\widehat{E}_{2^{k+1}}[f]\right|}\right)$, with $p, q, f(x)$, and $\widehat{E}_{n}[f]$ as in Table 1, for $k=1(1) 5$.

\begin{tabular}{||c||c|c|c|c|c||}
\hline$k$ & $j=0$ & $j=1$ & $j=2$ & $j=3$ & $j=4$ \\
\hline \hline 1 & 1.926 & 4.381 & 9.029 & 8.395 & 4.943 \\
2 & 2.044 & 4.019 & 6.175 & 9.892 & 14.490 \\
3 & 2.119 & 4.072 & 6.094 & 8.257 & 11.145 \\
4 & 2.163 & 4.129 & 6.113 & 8.123 & 10.168 \\
5 & 2.186 & 4.167 & 6.152 & 8.144 & 10.144 \\
\hline$\infty$ & 2.2 & 4.2 & 6.2 & 8.2 & 10.2 \\
\hline
\end{tabular}

TABLE 3. Errors $\widehat{E}_{n}[f]$ for the integral of Example 5.1 obtained with $n=2^{k}, k=1(1) 6$, and with the $K^{p, q}$ transformation. In column $j$, we have chosen $p=(j-\mu) /(1+\mu)$ and $q=j$. (Optimal $p, q$.

\begin{tabular}{||r||c|c|c|c|c||}
\hline$n$ & $j=0$ & $j=1$ & $j=2$ & $j=3$ & $j=4$ \\
\hline \hline 2 & $0.00 D+00$ & $1.26 D-03$ & $1.54 D-01$ & $3.22 D-01$ & $4.68 D-01$ \\
4 & $1.93 D-34$ & $1.51 D-06$ & $1.64 D-05$ & $2.87 D-04$ & $1.34 D-02$ \\
8 & $4.81 D-34$ & $1.00 D-11$ & $1.21 D-09$ & $2.33 D-08$ & $1.94 D-07$ \\
16 & $2.02 D-33$ & $1.25 D-21$ & $7.09 D-19$ & $6.76 D-16$ & $1.75 D-14$ \\
32 & $1.93 D-34$ & $9.63 D-35$ & $3.85 D-34$ & $9.03 D-31$ & $2.16 D-27$ \\
64 & $4.91 D-33$ & $9.63 D-35$ & $9.63 D-35$ & $1.93 D-34$ & $4.81 D-34$ \\
\hline
\end{tabular}


TABLE 4. Errors $\widehat{E}_{n}[f]$ for the integral of Example $[5.2$ obtained with $n=2^{k}, k=1(1) 6$, and with the $K^{p, q}$ transformation. In column $j$, we have chosen $p=(j-\mu) /(1+\mu)+0.1$ and $q=$ $(j-\nu) /(1+\nu)+0.1$. (Nonoptimal $p, q$.)

\begin{tabular}{||r||c|c|c|c|c||}
\hline$n$ & $j=0$ & $j=1$ & $j=2$ & $j=3$ & $j=4$ \\
\hline \hline 2 & $2.87 D-02$ & $1.91 D-01$ & $6.13 D-01$ & $8.67 D-01$ & $9.63 D-01$ \\
4 & $6.47 D-03$ & $6.37 D-04$ & $1.08 D-02$ & $1.40 D-02$ & $7.88 D-02$ \\
8 & $2.10 D-03$ & $1.20 D-04$ & $6.14 D-04$ & $5.30 D-04$ & $2.81 D-03$ \\
16 & $5.25 D-04$ & $8.58 D-06$ & $5.60 D-07$ & $9.35 D-07$ & $4.66 D-06$ \\
32 & $1.26 D-04$ & $5.09 D-07$ & $7.28 D-09$ & $2.21 D-10$ & $1.52 D-11$ \\
64 & $2.99 D-05$ & $2.98 D-08$ & $1.06 D-10$ & $7.91 D-13$ & $9.87 D-15$ \\
\hline
\end{tabular}

TABLE 5. The numbers $\rho_{p, q, k}=\frac{1}{\log 2} \cdot \log \left(\frac{\left|\widehat{E}_{2^{k}}[f]\right|}{\left|\widehat{E}_{2^{k+1}}[f]\right|}\right)$, with $p, q$, $f(x)$, and $\widehat{E}_{n}[f]$ as in Table 4, for $k=1(1) 5$.

\begin{tabular}{||r||c|c|c|c|c||}
\hline$k$ & $j=0$ & $j=1$ & $j=2$ & $j=3$ & $j=4$ \\
\hline \hline 1 & 2.149 & 8.225 & 5.826 & 5.957 & 3.611 \\
2 & 1.623 & 2.409 & 4.136 & 4.719 & 4.809 \\
3 & 2.000 & 3.806 & 10.099 & 9.146 & 9.236 \\
4 & 2.057 & 4.075 & 6.265 & 12.048 & 18.229 \\
5 & 2.079 & 4.094 & 6.105 & 8.125 & 10.586 \\
\hline$\infty$ & 2.05 & 4.05 & 6.05 & 8.05 & 10.05 \\
\hline
\end{tabular}

TABLE 6. Errors $\widehat{E}_{n}[f]$ for the integral of Example 5.2 obtained with $n=2^{k}, k=1(1) 6$, and with the $K^{p, q}$ transformation. In column $j$, we have chosen $p=(j-\mu) /(1+\mu)$ and $q=(j-\nu) /(1+\nu)$. (Optimal $p, q$.

\begin{tabular}{||r||c|c|c|c|c||}
\hline$n$ & $j=0$ & $j=1$ & $j=2$ & $j=3$ & $j=4$ \\
\hline \hline 2 & $5.00 D-02$ & $1.72 D-01$ & $5.81 D-01$ & $8.32 D-01$ & $9.25 D-01$ \\
4 & $1.67 D-03$ & $3.61 D-03$ & $6.45 D-03$ & $1.77 D-02$ & $8.16 D-02$ \\
8 & $5.81 D-05$ & $2.16 D-05$ & $4.71 D-04$ & $4.21 D-04$ & $3.04 D-03$ \\
16 & $2.51 D-06$ & $1.42 D-08$ & $5.65 D-08$ & $8.53 D-07$ & $3.71 D-06$ \\
32 & $1.04 D-07$ & $2.03 D-11$ & $4.22 D-16$ & $6.70 D-14$ & $4.42 D-12$ \\
64 & $4.23 D-09$ & $3.20 D-14$ & $1.69 D-30$ & $1.40 D-21$ & $4.88 D-25$ \\
\hline
\end{tabular}


TABLE 7. The numbers $\rho_{p, q, k}=\frac{1}{\log 2} \cdot \log \left(\frac{\left|\widehat{E}_{2^{k}}[f]\right|}{\left|\widehat{E}_{2^{k+1}}[f]\right|}\right)$, with $p, q$, $f(x)$, and $\widehat{E}_{n}[f]$ as in Table 6 , for $k=1(1) 5$.

\begin{tabular}{||r||c|c|c|c|c||}
\hline$k$ & $j=0$ & $j=1$ & $j=2$ & $j=3$ & $j=4$ \\
\hline \hline 1 & 4.906 & 5.577 & 6.494 & 5.558 & 3.503 \\
2 & 4.842 & 7.384 & 3.776 & 5.389 & 4.748 \\
3 & 4.534 & 10.571 & 13.025 & 8.948 & 9.675 \\
4 & 4.590 & 9.450 & 26.995 & 23.603 & 19.681 \\
5 & 4.622 & 9.309 & 47.825 & 25.508 & 43.040 \\
\hline$\infty$ & $4 \frac{2}{3}$ & $9 \frac{1}{3}$ & $\infty$ & $18 \frac{2}{3}$ & $23 \frac{1}{3}$ \\
\hline
\end{tabular}

TABLE 8. Errors $\widehat{E}_{n}[f]$ for the integral of Example 5.3 obtained with $n=2^{k}, k=1(1) 6$, and with the $K^{p, q}$ transformation. In column $j$, we have chosen $p=(j-\mu) /(1+\mu)+0.1$ and $q=$ $(j-\nu) /(1+\nu)+0.1$. (Nonoptimal $p, q$.)

\begin{tabular}{||r||c|c|c|c|c||}
\hline$n$ & $j=0$ & $j=1$ & $j=2$ & $j=3$ & $j=4$ \\
\hline \hline 2 & $7.99 D-03$ & $1.55 D-01$ & $3.04 D-01$ & $4.05 D-01$ & $4.64 D-01$ \\
4 & $3.22 D-03$ & $5.07 D-03$ & $1.86 D-02$ & $7.29 D-03$ & $2.02 D-02$ \\
8 & $7.99 D-04$ & $5.41 D-05$ & $6.70 D-05$ & $1.33 D-04$ & $1.17 D-03$ \\
16 & $1.74 D-04$ & $3.17 D-06$ & $1.41 D-07$ & $1.75 D-09$ & $1.97 D-08$ \\
32 & $3.69 D-05$ & $1.73 D-07$ & $1.95 D-09$ & $4.07 D-11$ & $1.37 D-12$ \\
64 & $7.70 D-06$ & $9.25 D-09$ & $2.65 D-11$ & $1.38 D-13$ & $1.15 D-15$ \\
\hline
\end{tabular}

TABLE 9. The numbers $\rho_{p, q, k}=\frac{1}{\log 2} \cdot \log \left(\frac{\left|\widehat{E}_{2^{k}}[f]\right|}{\left|\widehat{E}_{2^{k+1}}[f]\right|}\right)$, with $p, q$, $f(x)$, and $\widehat{E}_{n}[f]$ as in Table 8 , for $k=1(1) 5$.

\begin{tabular}{||r||c|c|c|c|c||}
\hline$k$ & $j=0$ & $j=1$ & $j=2$ & $j=3$ & $j=4$ \\
\hline \hline 1 & 1.310 & 4.932 & 4.029 & 5.796 & 4.518 \\
2 & 2.011 & 6.551 & 8.119 & 5.776 & 4.108 \\
3 & 2.199 & 4.095 & 8.893 & 16.216 & 15.862 \\
4 & 2.238 & 4.196 & 6.172 & 5.425 & 13.814 \\
5 & 2.262 & 4.222 & 6.203 & 8.200 & 10.212 \\
\hline$\infty$ & $2 \frac{2}{15}$ & $4 \frac{2}{15}$ & $6 \frac{2}{15}$ & $8 \frac{2}{15}$ & $10 \frac{2}{15}$ \\
\hline
\end{tabular}


TABLE 10. Errors $\widehat{E}_{n}[f]$ for the integral of Example 5.3 obtained with $n=2^{k}, k=1(1) 6$, and with the $K^{p, q}$ transformation. In column $j$, we have chosen $p=(j-\mu) /(1+\mu)$ and $q=(j-\nu) /(1+\nu)$. (Optimal $p, q$.

\begin{tabular}{||r||c|c|c|c|c||}
\hline$n$ & $j=0$ & $j=1$ & $j=2$ & $j=3$ & $j=4$ \\
\hline \hline 2 & $2.96 D-02$ & $1.39 D-01$ & $2.95 D-01$ & $4.03 D-01$ & $4.67 D-01$ \\
4 & $2.29 D-03$ & $1.97 D-03$ & $2.00 D-02$ & $1.29 D-02$ & $1.24 D-02$ \\
8 & $1.70 D-04$ & $4.99 D-06$ & $6.94 D-05$ & $3.76 D-05$ & $1.02 D-03$ \\
16 & $1.42 D-05$ & $5.99 D-08$ & $1.38 D-09$ & $5.23 D-09$ & $4.86 D-08$ \\
32 & $1.20 D-06$ & $4.34 D-10$ & $7.05 D-13$ & $2.62 D-15$ & $2.43 D-16$ \\
64 & $1.00 D-07$ & $3.08 D-12$ & $4.09 D-16$ & $1.19 D-19$ & $1.86 D-27$ \\
\hline
\end{tabular}

TABLE 11. The numbers $\rho_{p, q, k}=\frac{1}{\log 2} \cdot \log \left(\frac{\left|\widehat{E}_{2^{k}}[f]\right|}{\left|\widehat{E}_{2^{k+1}}[f]\right|}\right)$, with $p, q$, $f(x)$, and $\widehat{E}_{n}[f]$ as in Table 10, for $k=1(1) 5$.

\begin{tabular}{||r||c|c|c|c|c||}
\hline$k$ & $j=0$ & $j=1$ & $j=2$ & $j=3$ & $j=4$ \\
\hline \hline 1 & 3.697 & 6.141 & 3.883 & 4.971 & 5.237 \\
2 & 3.751 & 8.624 & 8.172 & 8.417 & 3.605 \\
3 & 3.584 & 6.378 & 15.621 & 12.812 & 14.352 \\
4 & 3.565 & 7.109 & 10.932 & 20.931 & 27.576 \\
5 & 3.574 & 7.138 & 10.751 & 14.420 & 36.928 \\
\hline$\infty$ & 3.6 & 7.2 & 10.8 & 14.4 & 25 \\
\hline
\end{tabular}

\section{ACKNOWLEDGEMENT}

This research was supported in part by the United States-Israel Binational Science Foundation grant no. 2004353.

\section{REFERENCES}

[1] M. Abramowitz and I.A. Stegun. Handbook of Mathematical Functions with Formulas, Graphs, and Mathematical Tables. Number 55 in Nat. Bur. Standards Appl. Math. Series. US Government Printing Office, Washington, D.C., 1964. MR0167642 (29:4914)

[2] P.J. Davis and P. Rabinowitz. Methods of Numerical Integration. Academic Press, New York, second edition, 1984. MR760629 (86d:65004)

[3] M. Iri, S. Moriguti, and Y. Takasawa. On a certain quadrature formula. Kokyuroku of Res. Inst. for Math. Sci. Kyoto Univ., 91:82-118, 1970. In Japanese. English translation in J. Comp. Appl. Math., 17:3-20, 1987. MR884257(88j:65057)

[4] P.R. Johnston. Application of sigmoidal transformations to weakly singular and nearsingular boundary element integrals. Intern. J. Numer. Methods Engrg., 45:1333-1348, 1999. MR:1699754 (2000c:74101)

[5] P.R. Johnston. Semi-sigmoidal transformations for evaluating weakly singular boundary element integrals. Intern. J. Numer. Methods Engrg., 47:1709-1730, 2000. MR.1750249

[6] N.M. Korobov. Number-Theoretic Methods of Approximate Analysis. GIFL, Moscow, 1963. In Russian. MR0157483(28:716)

[7] G. Monegato and L. Scuderi. Numerical integration of functions with boundary singularities. J. Comp. Appl. Math., 112:201-214, 1999. MR.1728460

[8] G. Monegato and I.H. Sloan. Numerical solution of the generalized airfoil equation for an airfoil with a flap. SIAM J. Numer. Anal., 34:2288-2305, 1997. MR1480381 (98f:45009) 
[9] M. Mori. An IMT-type double exponential formula for numerical integration. Publ. Res. Inst. Math. Sci. Kyoto Univ., 14:713-729, 1978. MR527197(81c:65012)

[10] T.W. Sag and G. Szekeres. Numerical evaluation of high-dimensional integrals. Math. Comp., 18:245-253, 1964. MR0165689 (29:2969)

[11] A. Sidi. A new variable transformation for numerical integration. In H. Brass and G. Hämmerlin, editors, Numerical Integration IV, number 112 in ISNM, pages 359-373, Basel, 1993. Birkhäuser. MR.1248416 (94k:65032)

[12] A. Sidi. Extension of a class of periodizing variable transformations for numerical integration. Math. Comp., 75:327-343, 2006. MR2176402 (2006g:41066)

[13] A. Sidi. A novel class of symmetric and nonsymmetric periodizing variable transformations for numerical integration. J. Sci. Comput., 31:391-417, 2007. MR2320555 (2008f:65046)

[14] A. Sidi. Asymptotic expansions of Gauss-Legendre quadrature rules for integrals with endpoint singularities. Math. Comp., 78:241-253, 2009. MR2448705

[15] A. Sidi. Further extension of a class of periodizing variable transformations for numerical integration. J. Comp. Appl. Math., 221:132-149, 2008. MR2458757

Computer Science Department, Technion, Israel Institute of Technology, Haifa 32000, ISRAEL

E-mail address: asidi@cs.technion.ac.il

$U R L:$ http://www.cs.technion.ac.il/〜asidi/ 